\title{
First-principles study of intermixing and polarization at the $\mathrm{DyScO}_{3} / \mathrm{SrTiO}_{3}$ interface
}

\author{
K. Rahmanizadeh, ${ }^{1}$ G. Bihlmayer, ${ }^{1}$ M. Luysberg, ${ }^{2}$ and S. Blügel ${ }^{1}$ \\ ${ }^{1}$ Peter Grünberg Institut (PGI-1) and Institute for Advanced Simulation (IAS-1), Forschungszentrum Jülich and JARA, \\ DE-52425 Jülich, Germany \\ ${ }^{2}$ Peter Grünberg Institut (PGI-5), Forschungszentrum Jülich, DE-52425 Jülich, Germany
}

(Received 17 November 2011; published 21 February 2012)

\begin{abstract}
The polar-to-nonpolar interface of $\mathrm{DyScO}_{3}$ and $\mathrm{SrTiO}_{3}$ was studied using density functional theory. Due to the polar discontinuity arising from nominally charged $\mathrm{DyO}$ or $\mathrm{ScO}_{2}$ layers, sharp interfaces induce a strong ferroelectriclike polarization in the $\mathrm{SrTiO}_{3}$, while in chemically mixed interfaces this discontinuity is avoided and no such polarization can be found. In both scenarios the interface remains insulating with only a small reduction of the band gap. Our calculations show that mixed interfaces are energetically more favorable than sharp ones, in agreement with recent experimental results that confirmed intermixing at these interfaces.
\end{abstract}

DOI: 10.1103/PhysRevB.85.075314

PACS number(s): 77.55.Px, 73.20.-r, 71.15.Mb, 68.37.Ma

\section{INTRODUCTION}

As a consequence of the reduced feature size in semiconductor technology, the $\mathrm{SiO}_{2}$ gate oxide of field-effect transistors become increasingly thinner. A continuation of this process leads to an increase of leakage currents, causing a waste of power consumption, and will at some point reach fundamental limits. Therefore, the search for high$\kappa$ dielectric materials to substitute $\mathrm{SiO}_{2}$ in silicon metaloxide-semiconductor field-effect transistors is of considerable importance. Currently, $\mathrm{HfO}_{2}$ is chosen as high- $\kappa$ gate oxide. However, the rare-earth scandates $\left(R \mathrm{ScO}_{3}\right.$, where $R$ is Dy, $\mathrm{Gd}$, and $\mathrm{La}$ ) were also proposed as alternative candidate materials for the replacement of $\mathrm{SiO}_{2}$ (Refs. 1-3).

Moreover, rare-earth scandates are a good choice as substrates for epitaxial growth of high-quality perovskite thin films. This type of substrate is frequently used for imposing strain on the paraelectric or ferroelectric thin film material. The strain can induce room-temperature ferroelectricity in $\mathrm{SrTiO}_{3}$, a material that is not ferroelectric at equilibrium volume at any temperature ${ }^{4}$ and it has been used to enhance the ferroelectric properties of $\mathrm{BaTiO}_{3}$ thin films. ${ }^{5}$ Recently, it has been discovered that a thin film of $\mathrm{EuTiO}_{3}$ grown epitaxially on a $\mathrm{DyScO}_{3}$ substrate shows the strongest ferroelectricity for any material that has been synthesized since 1966 and is simultaneously ferroelectric and ferromagnetic. ${ }^{6}$

In the last years, oxide interfaces have attracted considerable attention due to the emerging novel properties which do not exist in the corresponding parent bulk compounds. For example, joining the two band insulators $\mathrm{LaAlO}_{3}$ and $\mathrm{SrTiO}_{3}$ on the (001) faces can induce a wealth of new properties ranging from conductivity, to magnetism, and even to superconductivity. ${ }^{7}$ In a simple ionic model, $\mathrm{LaAlO}_{3}$ contains $\mathrm{LaO}$ and $\mathrm{AlO}_{2}$ with positive and negative net charges, respectively, while $\mathrm{SrO}$ and $\mathrm{TiO}_{2}$ layers are electrically neutral. As a consequence, an electric interface dipole is formed, which leads to a divergence of the electrostatic potential with increasing distance from the interface. This is claimed to be responsible for the many peculiar properties at the interface. On the other hand, intermixing at the interface, ${ }^{8,9}$ defects, ${ }^{10,11}$ or the formation of a ferroelectriclike polarization ${ }^{12}$ in the insulators are mechanisms that may contribute to prevent the divergence of the electric potential.
Recently, using high-resolution scanning transmission electron microscopy (HR-STEM) and electron energy loss spectroscopy (EELS), intermixing was reported for the DyScO${ }_{3} / \mathrm{SrTiO}_{3}$ interface. ${ }^{13}$ Dysprosium scandate exhibits the same polar layers as lanthanum aluminate: DyO layers have a nominal charge of $+1 e$ per formula unit, while $\mathrm{ScO}_{2}$ layers carry $-1 e$ charge per formula unit. In consequence of the off-stoichiometry at the interface, multilayers of $\mathrm{DyScO}_{3}$ and $\mathrm{SrTiO}_{3}$ were found to be electrically insulating.

Despite obvious similarities to the well-investigated $\mathrm{LaAlO}_{3} / \mathrm{SrTiO}_{3}$ interface, there are also considerable differences, as far as the structure of the parent compounds is concerned. While $\mathrm{LaAlO}_{3}$ crystallizes (as high-temperature phase) in an ideal cubic perovskite lattice, $\mathrm{DyScO}_{3}$ is of the orthorhombic $\mathrm{GdFeO}_{3}$ type (space group Pbnm). For the interface to $\mathrm{SrTiO}_{3}$ this implies that good lattice matching is achieved when the (110) surface of $\mathrm{DyScO}_{3}$ is interfaced with a $p(2 \times 2)$ unit cell of the $(001)$ surface of $\mathrm{SrTiO}_{3}$, while in the $\mathrm{LaAlO}_{3}$ case both (001) surfaces can be matched. These structural differences may lead to different stresses at the interfaces and influence the chemical composition of the interface layers.

Using first-principles calculations of $\mathrm{DyScO}_{3} / \mathrm{SrTiO}_{3}$ multilayers, we investigated two scenarios for avoiding the polar catastrophe, either by forming a mixed layer at the interface or, in a sharp interface, by formation of a polarization in the $\mathrm{SrTiO}_{3}$ substrate, finding a clear preference of the former mechanism with respect to the latter. We also provide a careful analysis of the energetics of these interfaces. This paper is organized as follows: After a description of the computational details in Sec. II, the bulk systems and their response to epitaxial constraints are investigated in Sec. III. The structural models for the sharp and mixed interfaces, their electronic structure and induced polarization are presented in Sec. IV, followed by summary and conclusion in Sec. V.

\section{COMPUTATIONAL METHOD}

The calculations have been performed using density function of theory (DFT) in the local density approximation (LDA) for the exchange-correlation potential. ${ }^{14}$ We employed the full-potential linearized augmented plane-wave (FLAPW) 
method $^{15}$ as implemented in the FLEUR code. ${ }^{16}$ The wave functions within the muffin-tin spheres were expanded in spherical harmonics with an angular momentum of up to $l=8$. In the interstitial region, plane waves with a reciprocal lattice vector cutoff $K_{\max }=4.0$ (a.u. $)^{-1}$ were included, whereas the convergence was tested up to 4.8 (a.u.) $)^{-1}$ for certain cases. For each compound, the muffin-tin radii $\left(r_{\mathrm{MT}}\right)$ were chosen as 2.51,2.50,1.91,2.10, and 1.30 a.u. for Sr, Dy, Ti, Sc and O, respectively. The $\mathrm{Sr} 4 s$ and $4 p$ states, Dy $5 s$ and $5 p$ states, and $\mathrm{Ti}$ and $\mathrm{Sc} 3 s$ and $3 p$ states were treated as local orbitals. In the nonmagnetic calculations, the eight $4 f$ electron of Dy were treated as core electrons, putting four spin-up and four spindown $4 f$ electrons in the core. In magnetic calculations, these states were treated as valence electrons using the LDA $+U$ scheme to account for the localized character of these states. ${ }^{17}$ As parameters we chose $U=10.8 \mathrm{eV}$ and $J=0.68 \mathrm{eV}$, values from the literature. ${ }^{18}$

Self-consistency was considered to be achieved when the total energy variation from iteration to iteration did not exceed $3 \times 10^{-5} \mathrm{eV}$ and forces on the atoms were converged to $50 \mathrm{meV} / \AA$. The reciprocal space was sampled by a mesh containing $40 \mathbf{k}$ points [corresponding to an $(8 \times 8 \times 8)$ Monkhorst-Pack mesh] in the irreducible wedge of the Brillouin zone for the cubic phases, and an equivalently dense k-point sampling for the supercells. For the structural optimization of the interfaces models, the lateral lattice constants were constrained to the values for $\mathrm{SrTiO}_{3}$, and the remaining degrees of freedom were optimized. This corresponds to the experimental situation, where the $\mathrm{SrTiO}_{3} / \mathrm{DyScO}_{3}$ layers were grown on a thick $\mathrm{SrTiO}_{3}$ film deposited on a $\mathrm{Si}$ (100) wafer. ${ }^{13}$

\section{BULK}

Experimentally, the structure of $\mathrm{DyScO}_{3}$ was determined to be of a distorted perovskite structure $\left(\mathrm{GdFeO}_{3}\right.$-type $)$ with orthorhombic symmetry and space group Pbnm. In our calculations, we determined the lattice parameters and internal coordinates of $\mathrm{DyScO}_{3}$ in a nonmagnetic and magnetic setup.

The results of both calculations, using LDA and LDA + $U$, are listed in Table $\mathrm{I}$, together with experimental data from Ref. 19. Both computational schemes lead to similar results, in good agreement with experimental values. While the lattice parameters are underestimated more strongly in the LDA $+U$ calculation (probably due to the contraction of the $4 f$ states), in this calculation the internal position of the Dy atom is in better agreement with the experimental values. Since in a structural and compositional analysis the strongest overall energy variation comes from the lattice parameters, we continue using the nonmagnetic setup. In this setup, the band gap was found to be $4.47 \mathrm{eV}$, in good agreement with previous calculations and about $24 \%$ smaller than the experimentally observed value. $^{18}$

Given a $\mathrm{SrTiO}_{3}(001)$ substrate, there are different models how an interface with $\mathrm{DyScO}_{3}$ can be formed. Considering the experimental $\mathrm{SrTiO}_{3}$ lattice parameter of $3.905 \AA$, a $c(2 \times 2)$ in-plane unit cell, that is, a centered, square cell containing two formula units, fits to the $\mathrm{DyScO}_{3}$ (001) surface if the $a$ and $b$ parameters are expanded or contracted by $1.5 \%$ and $3.5 \%$, respectively. In a constrained $c(2 \times 2)$ unit cell containing two formula units of $\mathrm{DyScO}_{3}$, where the
TABLE I. Computed and experimental values of the structural parameters for $\mathrm{DyScO}_{3}$ in the bulk orthorhombic Pbnm phase. The lattice constants $a, b$, and $c$ are given in $\AA$ and the internal parameters $x, y$, and $z$ are in units of these lattice constants. Experimental data were taken from Ref. 19.

\begin{tabular}{|c|c|c|c|c|}
\hline & $\begin{array}{c}a(\AA) \\
x\end{array}$ & $\begin{array}{c}b(\AA) \\
y\end{array}$ & $\begin{array}{c}c(\AA) \\
z\end{array}$ & $\begin{array}{l}\text { Deviation } \\
\text { to Expt. (\%) }\end{array}$ \\
\hline Expt. & 5.443 & 5.717 & 7.901 & \\
\hline Dy & 0.0174 & 0.0616 & 0.25 & \\
\hline $\mathrm{Sc}$ & 0.5 & 0.0 & 0.0 & \\
\hline $\mathrm{O}_{1}$ & 0.8738 & 0.4439 & 0.25 & \\
\hline $\mathrm{O}_{2}$ & 0.8114 & 0.1937 & 0.9341 & \\
\hline LDA & 5.386 & 5.673 & 7.838 & 0.9 \\
\hline Dy & 0.0161 & 0.0616 & 0.25 & 3.7 \\
\hline $\mathrm{Sc}$ & 0.5 & 0.0 & 0.0 & \\
\hline $\mathrm{O}_{1}$ & 0.8826 & 0.4495 & 0.25 & 1.1 \\
\hline $\mathrm{O}_{2}$ & 0.8077 & 0.1940 & 0.9380 & 0.3 \\
\hline $\mathrm{LDA}+U$ & 5.359 & 5.640 & 7.752 & 1.6 \\
\hline Dy & 0.0169 & 0.0619 & 0.25 & 1.7 \\
\hline $\mathrm{Sc}$ & 0.5 & 0.0 & 0.0 & \\
\hline $\mathrm{O}_{1}$ & 0.8815 & 0.4484 & 0.25 & 0.9 \\
\hline $\mathrm{O}_{2}$ & 0.8080 & 0.1934 & 0.9372 & 0.3 \\
\hline
\end{tabular}

rotation of the oxygen octahedra is the only degree of freedom for internal coordinates, we can optimize this rotation and vary the lattice constant, $c$, of $\mathrm{DyScO}_{3}$ perpendicular to the interface. The total energy of this simplified structure with respect to the orthorhombic ground state is very high, about $180 \mathrm{meV} /$ atom. We find a lattice constant $c=3.98 \AA$ and $\mathrm{a}$ rotation of the oxygen octahedra by $17.4^{\circ}$ around the $c$ axis, which corresponds to a Sc-O-Sc bond angle, $\alpha$, in the $(a, b)$ plane of $145.2^{\circ}$. A more flexible $c(2 \times 2)$ unit cell, containing four formula units, allows also for a tilting of the octahedra and a relaxation of the Dy positions. The energy of such a structure with the epitaxial constraint of the $\mathrm{SrTiO}_{3}$ lattice and an optimized lattice constant $c=7.88 \AA$ is then just $11 \mathrm{meV} /$ atom higher than the Pbmn ground state.

Another choice interfacing $\mathrm{DyScO}_{3}$ and $\mathrm{SrTiO}_{3}$, is to match the $(110)$ face of $\mathrm{DyScO}_{3}$ to a $p(2 \times 2)$ cell of the $(001)$ surface of $\mathrm{SrTiO}_{3}$, that is, a square cell containing four formula units of the material. This requires an in-plane compression of $1.1 \%$ and $1.2 \%$ along the [ $[\overline{1} 10]$ and [001] directions of $\mathrm{DyScO}_{3}$. Again, we investigate the energetics of a bulk unit cell containing eight formula units and constrained accordingly to estimate the cost of forming this interface. There are now more remaining degrees of freedom that have to be optimized, that is, the lattice constant in the [110] direction $(z$ direction in Fig. 1) that relaxes to $7.91 \AA$, the rotations and tiltings of the oxygen octahedra and the internal degrees of freedom of the Dy atoms. The relaxation of the oxygen octahedra can be characterized by specifying the angle of the Sc-O-Sc bond, $\alpha$, as indicated in Fig. 1. These angles are $\alpha_{x}=139.8^{\circ}$ and $\alpha_{y}=\alpha_{z}=143.5^{\circ}$ in the bulk and change only slightly due to the constraint, amounting to $140.83^{\circ}, 143.96^{\circ}$, and $144.14^{\circ}$ in the $x, y$, and $z$ direction, respectively. The resulting structure is energetically only $9 \mathrm{meV} /$ atom above the orthorhombic ground-state structure. 


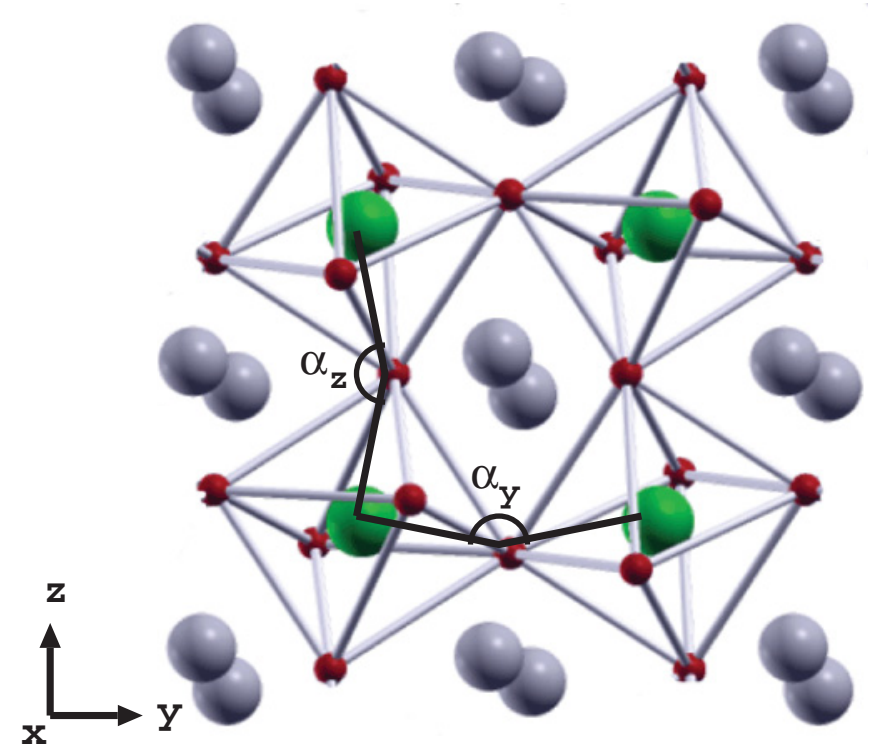

FIG. 1. (Color online) Relaxed $\mathrm{DyScO}_{3}$ bulk structure. The $(x, y)$ plane in this structure corresponds to the (110) plane and the $(y, z)$ plane to the (001) plane of the orthorhombic Pbnm structure. Gray spheres represent Dy, the small (red) spheres connected to octahedra are $\mathrm{O}$ atoms. In the center of these octahedra (green) spheres denote Sc atoms. Indicated are the Sc-O-Sc bond angles, $\alpha_{i}$, in the $z$ and $y$ directions. These angles are associated with the rotation of the octahedra around the $c$ axis $(x)$, while $\alpha_{x}$ describes the tilting of the octahedra with respect to this axis.

As compared to the above-mentioned DyScO${ }_{3}(001) / \mathrm{SrTiO}_{3}(001)$ interface, this structure is slightly more favorable, indicating a preference for a $\mathrm{DyScO}_{3}(110) / \mathrm{SrTiO}_{3}(001)$ interface model. This is also consistent with the experimentally determined HR-STEM images of this interface. ${ }^{13}$ It should, however, be kept in mind that the energetics of the real interface will be influenced also by other parameters that are not included in this bulk models. These aspects will be covered in the following.

\section{INTERFACES}

To model the interfaces between $\mathrm{DyScO}_{3}$ and $\mathrm{SrTiO}_{3}$, we used supercells of $m$ layers of $\mathrm{DyO}$ or $\mathrm{ScO}_{2}$ and $n$ layers of $\mathrm{SrO}$ or $\mathrm{TiO}_{2}$. These structures, denoted briefly as $(m, n)$ supercells, were then repeated periodically in the direction perpendicular to the interface. The in-plane lattice constant was kept fixed at $3.905 \AA$ for a $p(1 \times 1)$ unit cell. Both the simplified $\mathrm{DyScO}_{3}(001)$ and the more realistic $\mathrm{DyScO}_{3}(110)$ interface were studied. In the former case, a $c(2 \times 2) \mathrm{SrTiO}_{3}$ inplane unit cell is required, that allows already for a simulation of intermixing at the interface: For example, it is possible to create a $\left(\mathrm{Dy}_{0.5} \mathrm{Sr}_{0.5}\right) \mathrm{O}$ plane, with Dy and $\mathrm{Sr}$ arranged in a checkerboardlike fashion. The nominal charge in this layer is +0.5 , which allows a transition from the nonpolar to the polar material without creating a divergence of the potential in the latter: If the consecutive layer is stoichiometric $\mathrm{ScO}_{2}$ (nominal charge -1 ), as anticipated for $\mathrm{DyScO}_{3}$, the potential oscillates, however, around a constant value. Similarly, a $\left(\mathrm{Sc}_{0.5} \mathrm{Ti}_{0.5}\right) \mathrm{O}_{2}$ plane has a charge of -0.5 that, if followed by a DyO layer

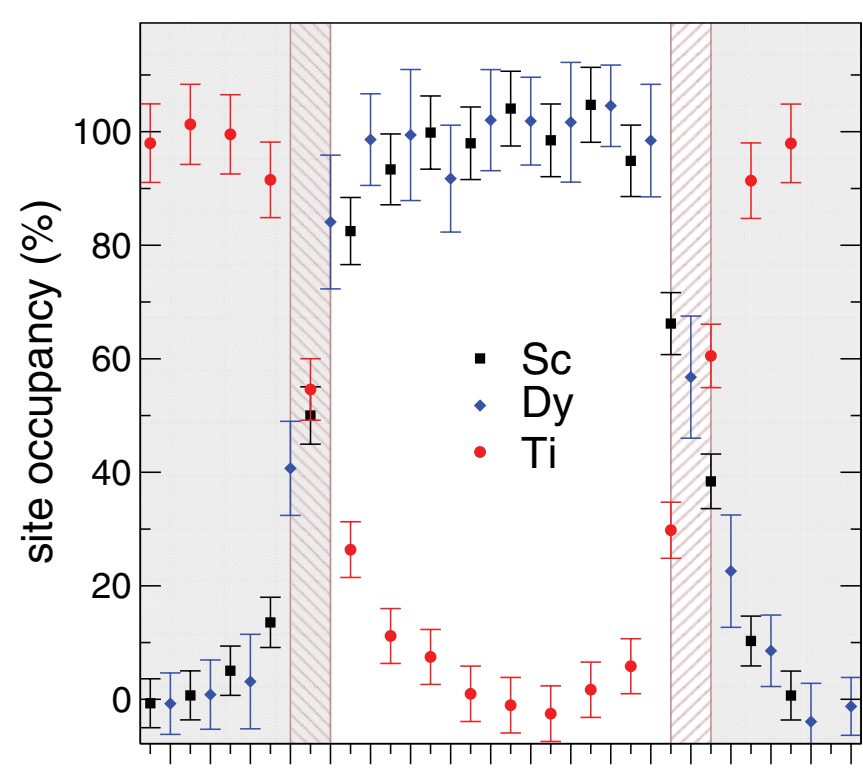

FIG. 2. (Color online) Experimentally determined concentration profile through a $\mathrm{DyScO} \mathrm{S}_{3} / \mathrm{SrTiO}_{3}$ interface. Long and short ticks on the $x$ axis mark $\mathrm{DyO}(\mathrm{SrO})$ and $\mathrm{ScO}_{2}\left(\mathrm{TiO}_{2}\right)$ layers, respectively. Gray/white areas indicate the regions of $\mathrm{SrTiO}_{3}$ and $\mathrm{DyScO}_{3}$ films, the hatched area marks the interface region. The values of the Dy/Sr layers have been obtained by quantitative evaluation of the HAADF intensities. The composition of the $\mathrm{Ti} / \mathrm{Sc}$ layers were extracted from the EEL spectra of the Ti $L_{23}$ and $\mathrm{Sc} L_{23}$ absorption edges.

$(+1)$, prevents the formation of a dipole that grows with the thickness of the scandate. To keep the stoichiometry, supercells with mixed interfaces have $\left(\mathrm{Dy}_{0.5} \mathrm{Sr}_{0.5}\right) \mathrm{O}$ and $\left(\mathrm{Sc}_{0.5} \mathrm{Ti}_{0.5}\right) \mathrm{O}_{2}$ layers at the two interfaces, while sharp interfaces contain $\mathrm{ScO}_{2} / \mathrm{SrO}$ and $\mathrm{DyO} / \mathrm{TiO}_{2}$ interfaces.

Experimentally, superlattices consisting of 20 DyScO $\mathrm{S}_{3} / \mathrm{SrTiO}_{3}$ double layers were grown by pulsed laser deposition. Alternating layers of $5 \mathrm{~nm}$ thickness of DyScO 3 and $\mathrm{SrTiO}_{3}$ were deposited on a 40-nm-thick $\mathrm{SrTiO}_{3}$ film on $\mathrm{Si}(001)$. High-resolution high-angle annular dark field (HAADF) images and EEL spectra were recorded as described in Ref. 13. The HAADF signal of the Dy and $\mathrm{Sr}$ columns is used to evaluate their composition across the interface. The Dy content is shown in Fig. 2. Ti and Sc concentrations of individual atomic layers are extracted from the EEL spectra of the corresponding $L$ edges. As a result an off-stoichiometric composition extending over about two interfacial layers is found (see Fig. 2). Moreover, we notice a similar distribution of Dy and Sc around the interface. While chemical intermixing was also observed at the $\mathrm{LaAlO}_{3} / \mathrm{SrTiO}_{3}$ interface, ${ }^{8}$ we note that in contrast to the present interface, the A cations of the polar material (La) diffuse deeper into the nonpolar substrate than the $\mathrm{B}$ ion $(\mathrm{Al})$. This difference, that might result from the difference in ionic radii between $\mathrm{La}$ and $\mathrm{Dy}$, can be responsible for the quite dissimilar conductive properties of these interfaces.

Compared to the experimental distribution, our model interface is very sharp, confining the off-stoichiometry to a single atomic layer. This implies the presence of an extra half charge per interface unit cell in this layers. In reality, due to the growth process, the interface is spread over a few layers. 

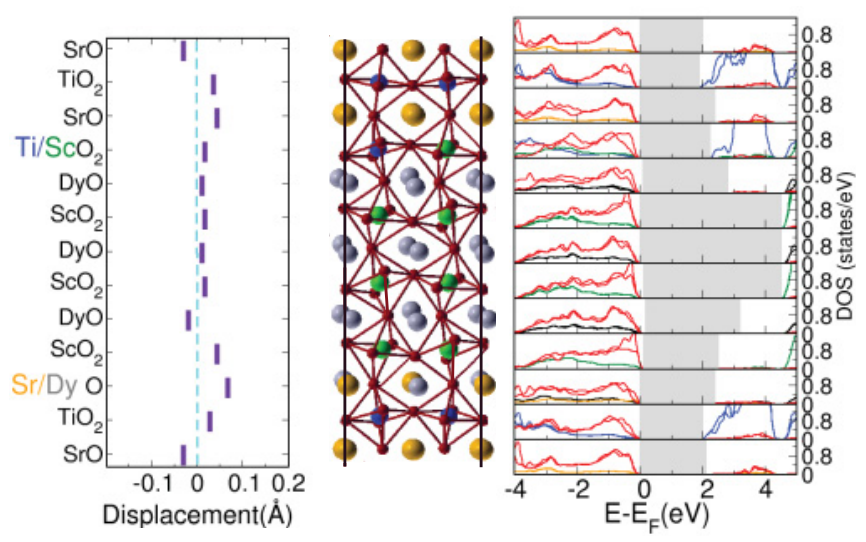

FIG. 3. (Color online) $\mathrm{DyScO}_{3}(110) / \mathrm{SrTiO}_{3}(001)$ mixed interface. In the left panel the average displacements of the cations (Dy, $\mathrm{Sr}, \mathrm{Sc}, \mathrm{Ti}$ ) with respect to oxygen ions in supercell are shown. The middle panel shows the structure with the (red) oxygen octahedra with Sc (green) or Ti (blue) atoms in the center and gray/light gray (golden) spheres representing the Dy and Sr ions. On the right we show the LDOS grouped by layers of the supercell, using the same color code as for the atoms. The gray shaded area indicates the band gap estimated from the LDOS of each layer. The data (displacement, structure, and LDOS) for each layer are aligned on the same vertical position.

Qualitatively, however, such a distribution will not change the conclusions drawn from our simulated mixed interface. We can also see that our approach to keep the stoichiometry of the individual compounds in the multilayer corresponds to the experimentally observed structure of the $\mathrm{DyScO}_{3} / \mathrm{SrTiO}_{3}$ multilayers, as can be seen from the concentration profile shown in Fig. 2: In the left interface (hatched area) a $\left(\mathrm{Sc}_{0.5} \mathrm{Ti}_{0.5}\right) \mathrm{O}_{2}$ layer is formed at the center, while on the right side a $\left(\mathrm{Dy}_{0.5} \mathrm{Sr}_{0.5}\right) \mathrm{O}$ layer can be observed.

To simulate the mixed $\mathrm{DyScO}_{3}(110) / \mathrm{SrTiO}_{3}(100)$ interface, we set up supercells containing four layers of each material and two interface layers with mixed stoichiometry. Since an in-plane $p(2 \times 2)$ unit cell is used, this corresponds to ten formula units of each material; that is, the $(5,5)$ supercell contains 100 atoms. A checkerboard-type arrangement of the atoms of different types is assumed in the mixed layers. The total energy of the setup was minimized by varying the out-of-plane lattice constant and the internal coordinates of the atoms. The whole structure was found to be insulating with a band gap of about $2.0 \mathrm{eV}$.

To investigate robustness of the results with respect to the the thickness of the $\mathrm{DyScO}_{3}$, we also simulated a structure containing six layers of $\mathrm{DyScO}_{3}$, four layers of $\mathrm{SrTiO}_{3}$, and the two mixed interface layers. In the notation introduced above, this is a $(7,5)$ supercell containing 120 atoms. A side view of this unit cell is shown in Fig. 3. The average displacement of the oxygen atoms with respect to the position of the cations (which, in a simple ionic model, is proportional to this layer's contribution to the polarization) is shown in the left panel of this figure. Note that we consider only displacements in the direction perpendicular to the interface here. It can be seen that only the mixed layers show a significant cation/anion displacement, while for the other layers bulklike values are
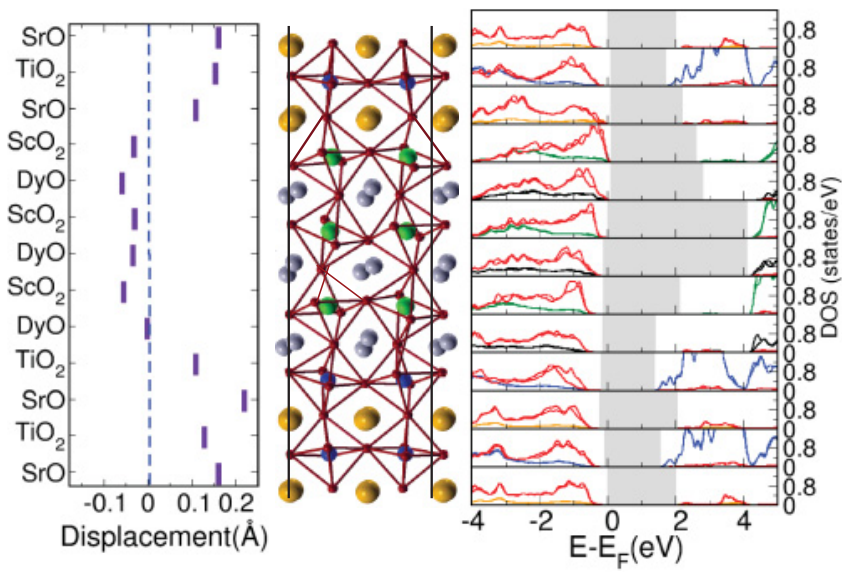

FIG. 4. (Color online) Same data as in Fig. 3, but for a DyScO $3(110) / \mathrm{SrTiO}_{3}(001)$ interface with a chemically sharp boundary. The induced polarization (left) ensures a band gap throughout the structure (right).

found. Plots of the local densities of states (LDOS) of the atoms in each layer are shown in the right panel of Fig. 3. Throughout the structure a band gap of at least $1.9 \mathrm{eV}$ is obtained, indicating again the insulating character of the interface. The insulating property of the structure is furthermore verified by inspection of the total DOS (not shown). This confirms the proposal that an off-stoichiometry at the interface helps to avoid the formation of a two-dimensional electron gas at the interface of a polar to nonpolar insulator. The same behavior was obtained for the simpler $\mathrm{DyScO}_{3}(100) / \mathrm{SrTiO}_{3}(100)$ interface.

In comparison to the mixed interfaces, we also set up supercells with sharp boundaries between $\mathrm{DyScO}_{3}(110)$ and $\mathrm{SrTiO}_{3}(100)$. Figure 4 shows a structure containing six layers of each compound, denoted as $(6,6)$ interface. Again, the structures were relaxed and we show the displacements of the cations with respect to the oxygen atoms in each layer. From the left panel of Fig. 4 significant and oppositely oriented polarizations in the two materials can be observed: On average, the oxygen anions move closer to the $\mathrm{DyO} / \mathrm{TiO}_{2}$ interface (nominally positively charged), while the cations relax toward the (nominally negative) $\mathrm{ScO}_{2} / \mathrm{SrO}$ interface. Since $\mathrm{SrTiO}_{3}$ is known as a highly polarizable material, the induced ionic polarization is larger in this material than in $\mathrm{DyScO}_{3}$. From the layer resolved densities of states (right panel in Fig. 4) it can be concluded that no metallic layer is formed at the interface also at the interfaces with sharp boundaries, similar to the mixed interfaces. A similar mechanism of induced polarization was proposed for the $\mathrm{LaAlO}_{3} / \mathrm{SrTiO}_{3}$ interface. ${ }^{10}$ Comparing interfaces of $\mathrm{LaAlO}_{3}$ with $\mathrm{SrTiO}_{3}$ grown on different substrates, a strong dependence of the electronic properties of the interface on the strain in the system was observed: ${ }^{20}$ This supports the idea that $\mathrm{SrTiO}_{3}$, depending on its strained state, can screen extra charges appearing at a polar interface. HR-TEM measurements actually confirmed the formation of cation to anion displacements around the interface. ${ }^{21}$

To compare the stability of the mixed and sharp interfaces, we also considered a system with eight layers of $\mathrm{DyScO}_{3}$ and four layers of $\mathrm{SrTiO}_{3}$, a $(8,4)$ supercell. Again, all internal coordinates and the lattice constant perpendicular to the 


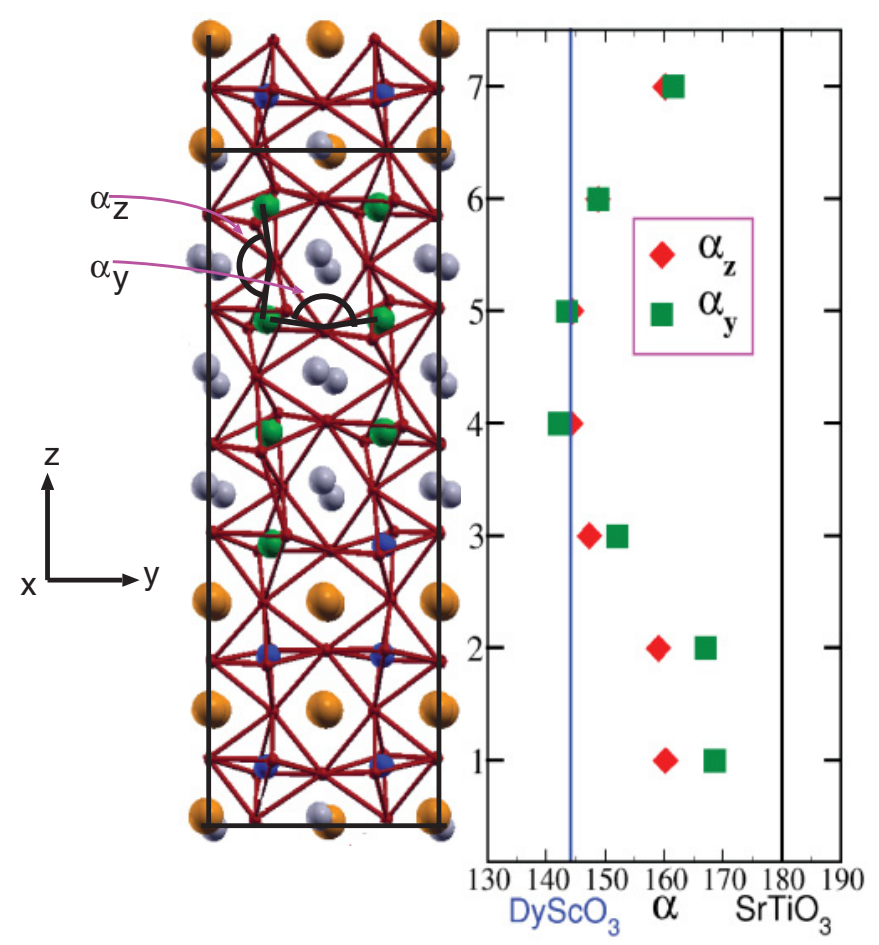

FIG. 5. (Color online) Detailed structure of the $\mathrm{DyScO}_{3}(110) / \mathrm{SrTiO}_{3}(001)$ mixed interface. The color code of the atoms corresponds to the one used in Fig. 3. The $B-\mathrm{O}-B$ bond angles $(B=\mathrm{Ti}, \mathrm{Sc})$, denoted as $\alpha_{y}$ and $\alpha_{z}$ are shown for the layers in the right panel. The lines at $144^{\circ}$ and $180^{\circ}$ represent bond angle in the bulk material of $\mathrm{DyScO}_{3}$ and $\mathrm{SrTiO}_{3}$, respectively.

interface were relaxed. Now, the energy gained by intermixing can be written as

$$
\Delta E^{(\text {mix }, \text { sharp })}=E^{(7,5)}-\frac{1}{2}\left(E^{(8,4)}+E^{(6,6)}\right) .
$$

Comparing these energies we find that the intermixed $\mathrm{DyScO}_{3}(110) / \mathrm{SrTiO}_{3}(100)$ interfaces are more stable by $65 \mathrm{meV}$ per $p(1 \times 1)$ in-plane unit cell. It should be mentioned that also the simplified $\mathrm{DyScO}_{3}(100) / \mathrm{SrTiO}_{3}(100)$ interface model gains a similar amount of energy by intermixing. We note in passing that from this calculations it can be seen that an increase of $\mathrm{DyScO}_{3}$ and decrease of $\mathrm{SrTiO}_{3}$ layers leads to a lowering of the band gap: As compared to $(6,6)$ supercells the $(8,4)$ structure has a $0.12 \mathrm{eV}$ smaller gap. Also in the $\mathrm{LaAlO}_{3} / \mathrm{SrTiO}_{3}$ system an induced polarization in $\mathrm{SrTiO}_{3}$ and a reduction of the band gap with thickness of the polar layer was observed. ${ }^{22}$
Finally, it is interesting to look at the structural aspects of the interfaces. Here we analyze the intermixed case, but similar features are found in the sharp interfaces, too. The $\mathrm{DyScO}_{3}$ structure is characterized by rotations of the oxygen octahedra around the $c$ axis and tiltings with respect to this axis. In Sec. III we introduced the Sc-O-Sc bond angles, $\alpha_{i}$, to characterize these distortions. Our calculations show how the rotation of the octahedra propagates into the bulk $\mathrm{SrTiO}_{3}$ through the interface: In Fig. 5 the angles $\alpha_{x}$ and $\alpha_{y}$ (both around $144^{\circ}$ in the ideal bulk structure), are shown as a function of the layers for the $(7,5)$ interface. A significant distortion of the ideal cubic perovskite lattice is observed, which does not decay to $180^{\circ}$ in our relatively thin $\mathrm{SrTiO}_{3}$ film. Similarly, in the experimental investigation of the $\mathrm{LaAlO}_{3} / \mathrm{SrTiO}_{3}$ interface, rotations of the oxygen octahedra were found to propagate several layers into the $\mathrm{SrTiO}_{3}$ films. ${ }^{21}$

\section{CONCLUSIONS}

The properties of bulk $\mathrm{DyScO}_{3}$ and $\mathrm{DyScO}_{3} / \mathrm{SrTiO}_{3}$ interfaces were investigated from first principles. The bulk structure of $\mathrm{DyScO}_{3}$ can be reasonably reproduced in the LDA or LDA $+U$ scheme for non-magnetic or magnetic calculations. We investigated the atomistic and electronic structure of the $\mathrm{DyScO}_{3}(001) / \mathrm{SrTiO}_{3}(001)$ and $\mathrm{DyScO}_{3}(110) / \mathrm{SrTiO}_{3}(001)$ interfaces using $c(2 \times 2)$ and $p(2 \times 2)$ in-plane unit cells. Chemically intermixed and sharp boundaries were considered. In both cases no metallic interface layers were found: While an off-stoichiometry naturally compensates additional charges at a polar-nonpolar interface, in case of the sharp interfaces a polarization of the lattice can remove these charges. We note here that this mechanism leads to a band insulator, in contrast to the Mott-insulating state that can be realized by introducing rare-earth oxide layers in $\mathrm{SrTiO}_{3}$ [Ref. 23]. As the $\mathrm{Ti}$ in our case is in a $\mathrm{d}^{0}$ state, effects of strong correlation are not considered here. If the formation of a conductive layer at the $\mathrm{DyScO}_{3} / \mathrm{SrTiO}_{3}$ interface is observed (as was achieved by variation of the growth conditions) this has indeed to be traced back to the presence of oxygen vacancies. ${ }^{24}$ From an energetic point of view, intermixing at the interface is clearly favorable. This is also confirmed by the experimental data on this system.

\section{ACKNOWLEDGMENTS}

The research leading to these results has received funding from the European Community's Seventh Framework Programme (FP7/2007-2013) under Grant Agreement No. NMP3LA-2010-246102. We gratefully acknowledge computing time from the Jülich Supercomputing Centre.
${ }^{1}$ C. Zhao et al., Appl. Phys. Lett. 86, 132903 (2005).

${ }^{2}$ K. H. Kim, D. B. Farmer, J.-S. M. Lehn, P. V. Rao, and R. G. Gordon, Appl. Phys. Lett. 89, 133512 (2006).

${ }^{3}$ S. Coh et al., Phys. Rev. B 82, 064101 (2010).

${ }^{4}$ J. H. Haeni et al., Nature (London) 430, 758 (2004).

${ }^{5}$ K. J. Choi et al., Science 306, 1005 (2004).
${ }^{6}$ J. H. Lee et al., Nature (London) 466, 954 (2010).

${ }^{7}$ M. Huijben, A. Brinkman, G. Koster, G. Rijnders, H. Hilgenkamp, and D. H. A. Blank, Adv. Mater. 21, 1665 (2009).

${ }^{8}$ P. R. Willmott et al., Phys. Rev. Lett. 99, 155502 (2007).

${ }^{9}$ L. Qiao, T. C. Droubay, V. Shutthanandan, Z. Zhu, P. V. Sushko, and S. A. Chambers, J. Phys. Condens. Matter 22, 312201 (2010). 
${ }^{10}$ M. S. Park, S. H. Rhim, and A. J. Freeman, Phys. Rev. B 74, 205416 (2006).

${ }^{11}$ G. Herranz et al., Phys. Rev. Lett. 98, 216803 (2007).

${ }^{12}$ R. Pentcheva and W. E. Pickett, Phys. Rev. Lett. 102, 107602 (2009).

${ }^{13}$ M. Luysberg, M. Heidelmann, L. Houben, M. Boese, T. Heeg, J. Schubert, and M. Roeckerath, Acta Mater. 57, 3192 (2009).

${ }^{14}$ L. Hedin and B. I. Lundqvist, J. Phys. C 4, 2064 (1971).

${ }^{15}$ E. Wimmer, H. Krakauer, M. Weinert, and A. J. Freeman, Phys. Rev. B 24, 864 (1981).

${ }^{16}$ [http://www.flapw.de].

${ }^{17}$ V. I. Anisimov, F. Aryasetiawan, and A. I. Lichtenstein, J. Phys. Condens. Matter 9, 767 (1996).
${ }^{18}$ M. Raekers, K. Kuepper, S. Bartkowski, M. Prinz, A. V. Postnikov, K. Potzger, S. Zhou, A. Arulraj, N. Stüßer, R. Uecker et al., Phys. Rev. B 79, 125114 (2009).

${ }^{19}$ B. Veličkov, V. Kahlenberg, R. Bertram, and M. Bernhagen, Z. Kristallogr. 222, 466 (2007).

${ }^{20}$ C. W. Bark et al., Proc. Natl. Acad. Sci. USA 108, 4720 (2011).

${ }^{21}$ C. L. Jia, S. B. Mi, M. Faley, U. Poppe, J. Schubert, and K. Urban, Phys. Rev. B 79, 081405 (2009).

${ }^{22}$ N. C. Bristowe, E. Artacho, and P. B. Littlewood, Phys. Rev. B 80, 045425 (2009).

${ }^{23}$ H. W. Jang et al., Science 331, 886 (2011).

${ }^{24}$ D. F. Li, Y. Wang, and J. Y. Dai, Appl. Phys. Lett. 98, 122108 (2011). 